\title{
Nochmals die Uebertragung der Lepra auf Thiere.
}

\author{
$\operatorname{Von}$ \\ Prof. Dr. Roberto Campana in Genua.
}

Wir haben uns bereits früher mit der Frage über die Uebertragbarkeit der Lepra auf Thiere beschäftigt ${ }^{1}$ ) und haben einige Experimente und Beobachtingen angestellt, welche uns zu ganz anderen Schlussfolgerungen als die von anderen Beobachtern angedenteten führten.

Wir wollen hier nicht wiederholen, was wir über diesen Gegenstand gesagt haben, und auch nicht anführen, was von anderen Beobachtern geäussert wurde; es genügt, einfach an die Namen $N$ is s $\theta \mathbf{r}^{2}$ ), Damsch ${ }^{9}$, Armauer Hansen ${ }^{4}$ ), Köbuor ${ }^{5}$ ), Melcheri) und

1) Note preliminari. Patologia della Lepra (Italia medica 1882. April). Alcune inoculazioni di noduli Icprosi (Archivio delle scienze mediche, hrsg. von Prof. Bizzozero, rol. VIII. Heft 1.-1883).

Della trasmissione della Iepra negli animali bruti (Clinica dermopatica e sifilopatica della $R$. Universitì di Genova, 1883. Heft I).

$\Rightarrow$ Weitere Beiträge zur Aetiologie der Lepra von Prof.Dr. A. Neisser. (Archiv für pathol. Anatomie u. Physiologie vol. 8'.-1881.)

$\left.{ }^{3}\right)$ Uebertragungs-Versuche von Lepra auf Thiere von Dr. O. Damsch. (Dasselbe Archiv vol. 92.-1883.)

4) Studien über Bacillus Leprae von Armauer Hansen in Bergen. (Dasselbe Archiv vol. 90. Heft III.-1882.)

$\left.{ }^{5}\right)$ Uebertragungs-Versuche von Lepra anf Thiere von Prof. H. Köbner. (Dasselbe Arichiv vol. 88.-1882.)

5) Cebertragung von Lepra auf Kaninchen von Melcher u. Ortmann. (Berliner klin. Wochenschr. 1885. Seite 293.) 
Ortmann ${ }^{1}$ zu erinnern, um zu begreifen, wie diese Frage erörtert wurde.

Wie den veröffentlichten Artikeln der genannten Autoren zu entnehmen ist, machen sich drei Meinungen über die Uebertragbarkeit oder Nicht-Uebertragbarkeit der Lepra auf Thiere das Feld streitig, und zwar folgende:

1. Diese Uebertragnng durch das Experiment wird beschränkt (Neisser) oder unbeschränkt (Damsch, Melcher und Ortmann) zugegeben.

2. Jede Uebertragung und auch jede Spur übertragener lepröser Reste wird in Abrede gestellt (Köbner und Andere).

3. Die wirkliche Uebertragung wird in Abrede gestellt, obgleich nicht selten an der Stelle, an welcher die Uebertragung ausgefübrt wird, Spuren übertragener Parasiten zu finden sind. (Campana.)

I.

Bevor wir untersuchen, wie sich diese Parasiten in den Thieren, denen sie eingeimpft werden, verbreiten, wird es gut sein, von ihrem gewöhnlichen Sitz in den Geweben, in denen sie sich aufhalten, zu sprechen; umsomehr als zwischen den Autoren, die sich mit diesem Gegenstande beschäftigen, noch lebhafte Discussionen stattfinden.

$\mathrm{Da}$ ich fünf Jahre lang beständig lepröse Individuen in der Klinik hatte, konnte jch die Lebensweise del Schistomyceten in ihren verschiedenen Phasen verfolgen. Während die Schüler sich übten, ausführliche Krankengeschichten lepröser Individuen zu sammeln, versäumte ich keine Gelegenheit, Untersuchungen über die Leprabacillen anzustellen.

Es ist nun das zweite Jahr, dass ich keine Leprakranken mehr in der Klinik habe, denn eine Verordnung der löblichen Verwaltung der Hospitäler verbietet die Zulassung von Leprakranken, sowie von Individuen, die mit gewissen anderen Krankbeiten (worunter die Hysterie) behaftet sind.

Indem ich die Widerrufung dieser Verordnung abwarte, benütze ich einstweilen das Material, über welches ich in meinem

1) Experimentelle Darm- und Lymphdrüsen-Lepra bei Kaninchen von Dr. R. Melcher u. Dr. P. Ortmann. (Berl. klin. Wochensclır. 1886. Nr. 9.) 
Dispensatorium, in meinem Laboratorium und in dem uuseres verehrten Rectors, des Herrn Professor Secondi, verfügen konnte.

Secondi, der sich seit einiger Zeit mit dem Kauterisiren der Hornhaut lepröser Individuen mittelst der Galvanokaustik beschäftigt, hat mich veranlasst, an einigen seiner Kranken Untersuchungen anzustellen.

In den Bindegewebszellen und in den Riesenzellen findet man die Leprabacillen; selten und nur in geringer Menge befinden sie sich ausserhalb der Zellen.

Um diese Thatsache richtig zu studiren, müssen folgende Bedingungen beachtet werden:

1. Nicht zu weit vorgeschrittenes Alter der Lepria beim Kranken und Wahl einer ebonfalls nicht alten Manifestation zur Anstellung der Beobachtung.

2. Eine Methode zur Färbung der Bacillen, welche das Protoplasma der za untersuchenden Gewebe nicht verändert.

3. Ausführung der Untersuchungen an Stücken, welche wo möglich nicht länger als drei Tage in absolutem Alkohol gelegen haben.

I. Es ist von Anderen, und namentlich von Noisser, Pellizzari und Maiocchi die Thatsache festgestellt worden, dass bei einer Lepra sehr alten Datums, deren Knoten runzelig und woich erscheinen, häufig keine Spur von Bacillen gefunden wird. - Und anatomisch kann man sagen, dass, wenn im kranken Gewebe die von körnig-fettiger Degeneration der Bindegewebs-Elemente abhängigen Veränderungen angetroffen werden, gewöhnlich keine oder nur sehr wenige Bacillen zu finden sind.

In Knoten einer Lepra neueren Datums, die sich hart anfühlen und von gleichmässiger Consistenz erscheinen, findet man alle bisher beschriebenen 'Veränderungen des leprösen Granuloms: Granulationszellen, die überall in ihrem Protoplasma mit den bekannten Bacillen gefüllt sind.

Tnd diese Bacillen befinden sich also im Innern der Granulationszellen, in den Riesenzellen: nämlich in jenen BindegewebsElementen, in denen sie vọr Allen Neisser gefunden hat, obgleich sie auch von Armauer Hansen bemerkt wurden, der sie jedoch erst nach den von $\mathrm{Neisser}$ gemachten Untersuchungen richtig beschrieben hat. 
Mit diesen Beobachtungen stimmen anch alle anderen neueren Forscher überein: Cornil, Touton in Wiesbaden, Thin in London, Belfield in Chigaco, sowie viele der schon genannten, und auch Dè Amicis, Peroncito, Primo Ferrari und Andere, welche ihre Meinungen nenerdings auf dern medicinischen Congress in Wiesbaden aufrecht erhielten. ${ }^{1}$ )

II. und III. Vor drei Jahren etwa ist jedoch von $\mathrm{Unn} \mathrm{a}^{2}$ ) diese Idee bekämpft worden, der zwar nicht in Abrede stellt, dass die Bacillen sich im Innern der Zellen befinden können aber der Meiuung ist, dass sie normaliter ausserhalb der Zellen, in den Lymphrăumen, liegen. Diese Idee wurde, wie man begreift, dadurch orzengt, dass er lepröse Stücke beobachtet hat, deren Zellen wegen des langwierigen Processes theilweise verändert waren, sowio dass von ihm hanptsächlich die Methode der Entfärbung mit Säuren angewendet wurde, durch welche das Zellprotoplasma verändert wird, und endlich, weil Stücke untersucht worden sind, die durch die lange Härtung alterirt waren.

In einer Mittheilung, die ich im Jahre 1883 der A ccade mia dei Lincei machte $\theta^{3}$ ), bemerkte ich, dass, "wenn jene Hautstück $\theta$ nicht nach der Ehrlich'schen Methode behandelt, sondern einfach mit Gentianaviolet gefürbt und darnuf in Wasser, Alkohol und Nelkenöl präparirt, in Canada-Balsam eingeschlossen wurden, .... mit einem Mikroskop von 800 D. Vergrösserung, die Existenz jener Bacillen und Körnchen wahrgenommen werden konnte; aber diese zeigten sich in dem Protoplasma jeder Zelle, ein Protoplasma, das nach der Ehrlich'schen Methode fast gänzlich aufgelöst wurde." Wie man leicht begreifen wird, hat jene Präparir-Methode nicht die desorganisirenden Wirkungen, welche bei der Ehrlich'schen Methode dem Gebrauch starker Säuren zuzuschreiben sind. Bemerken möchte ich jedoch, dass ich schon seit längerer Zeit mich nicht mebr des Nelkenöls zum Aufhellen der mikroskopischen Stücke bediene, und zwar wegen dessen schlechter Wirkung auf die Färbung.

1) Verhandlungen des Congresses für innere Medicin in Wiesbaden, 1886.

') Unna, Mon. f. Dermatol. und Syph. 188 s.

$\left.{ }^{3}\right)$ Osservazioni sulla Medicazione locale della Lepra. (Accademïa dei Lincei, anno CCLXXX.) 
Bezüglich der Art des Präparirens der Leprabacillen erwähne ich ferner, dass ich mich auch der Gram'schen Methode, der von Koch ursprünglich für die Tuberculose vorgeschlagenen Methode, sowie der von Lustgarten zur Untersuchung der Syphilisbacillen ersonnenen Methode bedient habe.

Da ich mich eine Zeit lang in Gesellschaft des Dr. Giletti befand, der sich längere Zeit mit der Untersuchnng des sogenannten Syphilisbacillus beschäftigt hat, wollten wir die Wirkungen der letztgenannten Färbungsmethode beim Leprabacillus feststellen; und ich muśs sagen, dass deren Resultate durchaus lobenswerth sind und dass diese Methode nicht weniger überzengend ist, als was ich mit der Koch'schen und Ehrlich'schen Methode beobachtet habe.

$\mathrm{Ja}$, es muss in dieser Hinsicht ein Vortheil der Technik erwähnt werden, den diese letztere Methode vor den anderen voraus hat. Während nämlich die Ehrlich'sche Methode ein wenig die Hornschichte und einen Theil der Talgdrüsen gefärbt lässt, was mitunter Zweifel hervorruft, ob in diesem Theile überhaupt Bacillen vorhanden sind, erzengt die Lustgarten'sche Methode') die vollständige Entfürbung der Stücke und lässt nur die Bacillen gefärbt. Nunwohl, mit dieser Methode haben wir beide keine Spur von Bacillen in Zusammenhang mit den Talgdrüsen finden können, wie dies $B a b e s^{2}$ ) gefunden zu haben behauptet. Man beachte, dass es nichts Seltenes ist, einen Bacillus ausserhalb der dem Leprabacillns eigenen Gebiete zu finden, und zwar in Folge der während des Präparirens erfolgten mechanischen Uebertragung desselben; dass sich aber solche wirklich in den Talgdrüsen befinden sollen, wird durch die Lustgarten'sche Methode entschieden verneint.

\section{II.}

Wir wollen jetzt anf einige andere Besonderheiten der Biologie und Morphologie des Leprabacillus zu sprechen kommen, um uns dann mit den von uns erwäbnten neuen Experimenten zu beschäftigen.

Wie die numerische Menge der Leprabacillen in directem

1) Lustgarten, Die Syphilisbacillen. (Jahrb. d. Gesellsch. d. Aerzte in Wien 1885, mit 4 chromolithogr. Tafeln.)

$\left.{ }^{2}\right)$ Babes. (Soc. de biologie, Mars 1883, und Arch. de physiologie, Juillet 1883.) 
Verhältniss zur Dauer der Lepra abnimmt, so lässt dieser Bacillus seine oberflächlichen Spuren in den Geweben in den letzten Perioden des von ihm verursachten Leidens zurïck.

Im Allgemeinen kann man sagen, dass, während man in einer jungen Granulationszelle eines Knotens neueren Datums die Bacillen in Haufen wahrnimmt, in einer Zelle alten Datums solche nur in geringer Menge, bis zum gänzlichen Verschwinden beobachtet werden.

Dieses erklärt den Unterschied, den man zwischen den Figuren verschiedener Autoren beim Vergleichen derselben findet: in einigen sieht man hier und dort wenige Bacillon, die einander nicht gleichen; in anderen wiederum sieht man deren viele und deutlicher und gleichartiger.

In den rückständigen Infiltrationen findet man oft keine Bacillen mehr. Die Zellen lassen sich mit den gebränchlichen Methoden leicht färben, aber in ihrem Innern lassen sie keine Spur von Schistomyceten erkennen; nur ab und zu bemerkt man grössere Zellen, die sich wenig mit Carmin, Hämotoxylin u. s. w. färben; bei diesen bemerkt man im Protoplasma ganz kleine Körnchen, welche wie die wahrscheinlichen Sporen aussehen, die man im Innern der Bacillen bemerkt; dieselben verändern sich nicht bei der Behandlung mit Aether, Kali cansticum, sie werden auch nicht kleiner durch die Furchung und nicht grösser durch die Conglomeration, wie dies bei Fettsubstanzen stattfindet. Doch lassen sich diese Sporen oder Coccen (Coccothrix Unnae) nicht oder doch nur wenig mit der Ehrlich'schen Methode färben, besser jedenfalls mit der nenen Unna'schen Methode.

Ich dabe Gelegenheit gehabt, alle Organe lepröser Individuen zu studiren; denn dies ist für mich ein Arboitsfeld, wenn es mir an anderen Arbeiten fehlt. In dieser Hinsicht berufe ich mich auf die bei anderen Gelegenheiten veröffentlichten Arbeiten. Hier bemerke ich nur, dass die Beobachtung lepröser Tumoren alten Datums, in denen sich keine Bacillen mehr oder doch nur wenige vorfinden, die Idee begünstigt, dass diese Bacillen sich in den Zellen und nicht ausserhalb derselben in Zooglöen befinden, insofern als die Kargheit der parasitischen Elemente diese Untersuchung erleichtert. Ausserdem befinden sich - die rückständigen Coccen, die sich erst nach 12 Stunden mit-der Ehrlich'schen 
Methode färben, im Innern der Zellen und nicht ausserhalb derselben. Enrwähnt sei hier, dass jch Veranlassung nahm, die Untersuchungen auch bezüglich der leprösen Nenritis zu wiederholen, wie ich andernorts mitgetheilt hab ${ }^{1}{ }^{1}$ )

\section{III.}

In aller Kürze sei hier die klinische und anatomische Geschichte des leprösen Individuums mitgetheilt, von dessen Leichnam die Stücke zu den Untersuchungen genommen wurden.

Es ist ein Jüngling von etwa 15 Jahren, welcher seit 5 Jahren an dieser Krankheit leidet; er zeigte erysipelasartige Flecken auf verschiedenen Stellen der Hautoberfläche, knotige Ausschläge im Gesicht und auf den Gliedern, Wasserbläschen auf den Gliedern, Symptome des wiederkehrenden Fiebers.

Jetzt beobachtet man diffuse und bedeutende Haut-Verdickungen am Gesicht, besonders an der Nase, den Wangen, der Stirn, welche seine Physiognomie sehr verunstalten; das Gesicht ist glatt, gelblich-braun, glänzend. Auf dem Rumpf zeigtt er mehrere bräunlich-rosenfarbige, glatte Knoten von verschiedener Grösso, von der Grösse einer kleinen Haselnuss bis zu der einer SousMünze, weich beim Anfühlen.

Auf den Gliedern beobachtet man folgende Modificationen: Die Haut der Handfäche ist sehr dünu, dagegen ist das subcutane Gewebe verdickt und härter als im Normalzustande; die darunter liegenden Muskeln sind an Umfang vermindert, sowohl in der Gegend des Handballens als in der des kleinen Fingers. Anch die Zwischenknochenmuskeln auf der Rückseite der Hände sind an Umfang vermindert.' Auf den Ober- und Vorderarmen papulöse Knötchen von verschiedener Grösse und ron derselben Art wio die auf dem Rumpf beobachteten. Auf den unteren Gliedern: zerstrente Knoten, wie die auf dem Rumpfe, auf den Schenkeln; magere Beine mit dürrer, schlaffer, nicht sehr dicker Hant; Unterhautschicht dicker. als im Normalzustande; Muskeln an Umfang vermindert. Verminderte Muskel-Contractilität in den Gliedern. Alterirter oder verminderter Gefühlssinn auf denselben Gliedern und auf allen Ausschlagistellen; verminderte thermische, elektrische

1) Enciclopedia medica italiana, hrsg. von Vallardi: Artikel Erpete. 1883. 
und Schmerz-Sensibilität. Chromocytometrie (Chromocytometer von Bizzozero) im Durchschnitt 160. Die anderen Alterationen lassen wir der Kürze halber hier weg.

Diagn ose. Lepra tuberculosib mit Nébildungen der Haut, sowie der Schleimhaut der Nase, des Gaumens und des Kehllsopfs; chronische lepröse Neuritis; leprösc Milz- und Leber-Anschwellıng; wiederkehrender lepröser Pemphigus; Fieber.

Die anatomische Diagnose stimmte mit der oben mitgetheilten klinischen überein, es wurden ausserdem nur noch Ulcerationen des Dünndarmes constatirt, von denen wir bei wiederkelurenden Darmkatarrben Zeichen erbalten hatten. .

Experimente. Einige Forscher haben, wie wir vorhin erwähnten, behauptet, dass die Lepria auf Thiere übertragen werden könne.

Ich machte vor einiger Zeit Experimente, mit denen ich zu zeigen suchte, dass bei Uebertragung von Leprastücken diese nach einer gewissen Zeit absorbirt werden und an der betreffenden Stelle keine Spur von sich zurücklassen; und dass, so lange die vollständige Aufsangung derselben nicht stattgefunden hatte, in den um die betreffende Stelle liegenden Geweben Spuren von Entzündung mit hier und dort zerstreuten und von der Zerstörung des übertragenen Stückes herrührenden Leprabacillen gefunden werden konnten.

Diese Idee kann ich jetzt durch neue Experimente befestigen, und sind dieses die folgenden:

Ich habe Stücke von der Haut und von anderen Organen mit leprösen Infiltrationen genominen. Dieselben habe ich oft untersucht; um nach dem Leprabacillus zu forschen, ehe ich sie zu den Experimenten verwendete; und wie gesagt, anfangs machte ich diese Untersuchung nur mitunter, später aber beständig; denn einige Experimente, welche jch nach dem ersten machte, musste ich im Stiche lassen, weil jch versäumt hatte, die betreffenden Stïcke vorber zu prüfen, und die Untersuchungen mir nicht die Resultate gaben, welche ich anfangs erhalten hatte.

Die von mir ausgeführten Experimente sind die folgenden:

Ich nahm lepröse Stücke, hüllte sie in Celloidin ein und zerschnitt sie dann in Sectionen. Hievon untersuchte ich einige nach der Ehrlich'schen Methode, die anderen wusch ich, nachdem 
ich sie vorher mit Alkohol und Aether vom Celloidin befreit hatte, mit Chlornatrium-Wasser $(0,75 \times 100)$ durch Wärme sterilisirt, und brachte sie nachher in einen gehörig sterilisirten Glasmörser. Ich reducirte Alles zu einem dünnen Brei, den ich dann mit dem sogenannten Chlornatrium-Wasser verdünnte, bis er das Aussehen einer Amygdalin-Emulsion hatte; ich injicirte mit einer geeigneten Pravaz'schen Spritze von ungefähr 5 Gramm Inhalt diese Flüssigkeit in die Bauchfellhöhle und machte gewöhnlich eine andere Injection in das subcutane Gewebe einiger Meerschweinchen.

Erwähnt sei, dass sowohl die Haut der Thiere wie die Instrumente je nach den Fällen mit Sublimat oder Carbolsäure sterilisirt wurden, und dass die leprösen Gewebsstücke, deren ich mich bediente, drei Jahre lang in absolutem Alkohol aufbewahrt wordon waren.

Nach 24 Stunden erzeugte ich in den Thieren, die ich injicirt hatte, mittelst 2-6stündiger Ligatur der Glieder, Oedeme.

Mit kleinen Stichen brachte ich die wässerige Flüssigkeit der Oedeme heraus und untersuchte sie mit dem Mikroskop, indem ich einen Tropfen derselben auf eine Glasplatte that, ihn trocknete und nach der Ehrlich'schen Methode behandelte.

Bei den ersten Experimenten fand ich immer zerstreute Bacillen und Sporen und, wie natürlich, einige Bacillen isolirt, andere vereinigt und wieder andere, welche ein Stück des Zellprotoplasmas, von dem sie durch das Stampfen nicht getrennt worden waren, mit sich trugen. Eine Zeit lang fehlte bei zwei Thieren jedes Resultat; aber die Ursache hievon war, dass wir eine Gewebs-Emulsion injicirt hatten, in welcher sich keine Bacillen befanden, wie wir dieses später feststellten.

Andere später gemachte Versuche haben stets positive Resultate ergeben; nämlich Anwesenheit von Bacillen, von Sporen und von Vereinigungen mehrerer Bacillen, frei oder leukocytenähnlich übereinandergehäuft.

Bei dieser Methode war jedoch ein Uebelstand: oft traten die Färbungen nicht deutlich hervor.

Uebrigens haben wir nicht nur die wässerige Flüssigkeit untersucht, sondern auch Gewebsschnitte; und diese haben uns viel klarere Resultate gegeben, indem wir die Schnitte wach 
der mehrmals erwälnnten Ehrlich- und Koch'schen Methode behandelten.

Wir wollten jedoch die Methode noch mehr vereinfichen.

Statt einer ganz farblosen Masse von Bacillen, injicirten wir eine gefärbte. Wir gingen in folgender Weise zu Werke: die Schuitte wurden nach der Ehrlich'schen Methode gefürbt, sodann zu Brei reducirt und in Form der gewöbnlichen Emulsion injicirt.

Mit dieser Methode haben wir folgende Resultate erhalten: Nach 24 Stunden erzeugten wir bei zwei Meerschweinchen (ein ziemlich grosses Männchen und ein kleines Weibchen) die gewöhnlichen Oedeme.

Die fast klare, durchsichtige Flüssigkeit derselben hat uus bei der Forschung nach dom Leprabacillus folgende Resultate gegeben:

Ohne jede andere Behandlung, mit dem Mikroskop untersucht, zeigte sie rosagefärbte Körnchen. Doch trat deren Fürbuur nicht klar hervor.

Wenn wir dagegen ron dieser Untersuchung'swoise zur Ehrlich'schen Methode zurückkehrten und die Präparate nach dor letzteren Methode färbten, traten die Sporen und Bacillen deutlicher hervor.

In dem dem linken Schenkel eines Meerschweinchens entnommenen serösen Material, nachdem in diesem Schenkel besagto Emulsion injicirt worden war, waren die Bacillen dentlich und gut gefärbt und zeigten sich dieselben isolirt oder: in leukocytenähnlichen Zellen aufeinandergehäuft.

Doch waren die anf diese Weise gefundenen parasitischen Elemente nicht in numerischem Verhältniss zu den injicirten.

Eine Untersuchung mit besseren Resultaten war jene, welche mit in absolutem Alkohol gehärteten und dann geprüften Orgaven gemacht wurde.

Nachdem diese Stücke mit dem gewöhnlichen Mikrotom in Sectionen zerlegt und gefärbt oder auch nicht gefärbt worden waren, haben wir folgende Resultate erhalten:

Hier und dort, den Lymphräumen entlang -zerstrent, sah man gefärbte Sporen oder Bacillen, doch in geringer Anzahl und von rosenrother Farbe. Viele Endothelialzellen trugen solche anf ihrer Oberfläche und hatten jenes Aussehen, auf welches ich schon 
in einer früheren Arbeit hingewiesen habe, wo diese Zellen Köruchen von Anilinblan, welches zur Injection verwordet wurde, $a b-$ sorbirt hatten.

In einer Section von Bindegewebe beobachtete man nicht nur die Endothelialzellen mit Bacillen im Iuneru, sondern man gewabrte auch hier und dort Leukocyten; welche einen leichten Grad von Infiltration bildeten und welche anch am peripheren Theile Bacillen zeigten.

Die Ursache dieses reichlicheren Vorbandenseins von Leukocyten ist der Stase zuzuschreiben, welche wir vorher in den Gliedern dieses Meerschweinchens erzeugt hatten, sowie traumatischen Irritationen, die durch den Bruch eines Knöchelchens an verschiedenen Stellen hervorgerufen wurden.

Der erwähnte Bruch war subcutan und die Thiere wurden in einem sehr reinen Zimmer gehalten nnd täglich zweimal mit einer Sublimatlösung $(1 / 1000)$ gewaschen; um zufällige Infectionen zu vermeiden.

Die Meerschweinchen, die wir benützten, boten keine Anzeichen der Tuberculose oder der Psorospermie dar. Wir hatten allerdings einige, welche dieses letztere Leiden offenbarten, doch haben wir dieselben nicht benützt.

Wir wollen diese unsere Untersuchungen nicht mit dem vergleichen, was Andere gefunden haben, da mit mebreren derselben die objectiven Resultate übereinstimmen, wohingegen allerdings deren Auslegung differirt. Nur möchten wir auf einige von den Herren Dr. Ortmann und Dr. Melcher berichtete klinische Besonderheiten hinweisen. Wir bemerken zuvor noch Folgendes.

Zu unseren Experimenten haben wir (jetzt und früher) frisch ausgeschnittene und vor längerer Zeit ausgeschnittene und 2-3 Jahre lang in absolutem Alkohol anfbewahrte Stücke benützt.

Es ist bekannt, dass der absolute Alkohol mit seiner lange anhaltenden wasserentziehenden Wirkung selbst Organismen, die widerstandsfähiger sind als ein Leprabacillus, das Leben raubt.

Wir bezweifeln nun nicht die Genauigkeit der Beobachtungen Melcher's und Ortmann's, dass in den im Blinddarm erzeugten Tumoren und in den Anschwellungen der Lymphdrüsen des Banches und des Halses Leprabacillen gefunden worden sind; wir glauben aber, dass die groben Manifestationen, welche der Lepra 
zugeschrieben werden, sich auf andere Zustände beziehen müssen (gewöhnliche Irritationen, Tuberculose, Psorospermie u. s. w.). In der That könnten jene Alterationen, derentwegen einige der neoplastischen Bauchknoten ein Flüssigkeitsmaterial enthielten, das wie aus einer Cyste geleert werden kann, klinisch nicht erklärt werden; und einige jener hyperplastischen Drüsen sind weich geworden wie die Knoten des Darmkanals (Berl. klin. Woch. 1886, Seite 136), während doch bekannt ist, dass bei der Lepra das Stadium der Erweichung sämmtlicher Lepraknoten sebr selten ist; dass dagegen Phasen nekrotischer Erweichung ausgedehnter gesunder oder kranker Gewebsstrecken festgestellt werden, entweder in Folge des veränderten trophischen Einflusses der Nerven, der Glieder u. s. w. oder in Folge wiederkehrender acuter Perioden des Leidens (sogen. Lepratyphus), bei welchem die allgemeinen Symptome einer schweren acuten Infectionskrankheit nicht fehlen. Und es scheint nicht, dass es sich um diese Krankbeitsform bei den in Frage stehenden Thieren bandeln könne, denn es fehlen Angaben über allgemeine Störungen (Fieber u. s. w.).

\section{Schlussfolgerung.}

Diese zweite Serie ron Beobachtungen führt uns zu folgenden Scblussfolgerungeu.

Der Drebpunkt, auf den sich alle Diejenigen stützen, welche die Uebertragbarkeit der Lepra auf Thiere zugeben, ist nicht die klinische Beobachtung; denn von den Autoren selbst wird behauptet, dass das Leiden mit einer Tuberculose verwechselt werden könne. Der Haupt- und Drehpunkt ist also die Demonstration des Leprabacillus mit den Merkmalen, welche ihn vom Bacillus der'Tuberculose unterscheidbar machen: nämlich 1. leichte Färbbarkeit mit fast allen Methoden und besonders mit der Ehrlich'schen in wenigen Minuten, im Gegensatze zum Bacillus der Tuberculose, der entweder Erwärmung oder ein 24 Stunden Ianges Eintauchen in anilinisirte und gefärbte Flüssigkeit orforderlich macht; 2. die grosse Menge dieser Parasiten bei der Lepra, im Gegensatze zur geringen Menge bei der Tuberculose.

Alles dieses nun kann erklärt werden - nicht durch die Annahme, dass in den Thieren wirklich Lepra erzeugt wurde, sondern damit, dass die Leukocyten sich die Parasiten aneignen, 
die nun wie fremde Körnchen in den lymphatischen Geweben jenes Organismus schwimmen, in welchen sie injicirt oder inoculirt worden sind, wie bei den Thieren, mit denen ich experimentirt habe, bei Hühnern, Meerschweinchen und Kaninchen.

Die Meerschweinchen, welche ich zu meinen letzten Untersuchungen benützte, wurden 1 bis 8 Tage am Leben erhalten; denn jetzt wollte ich nicht mehr beweisen, dass sich Bacillen in den Thieren lange Zeit nach der Uebertragung eines leprösen Materials befinden, ohne dass eine Erzengung des Leidens stattfinde, was ich schon früher gemacht hatte, sondern einfach, dass nach den Injectionen eines sicherlich getödteten, Leprabacillen tragenden Materials, davon Spuren an Stellen gefunden werden können, die weit vom Orte der Injection entfernt sind und an denen Reizungs-Ursachen Zufluss von Leukocyten erzeugt haben. 